\title{
Infinite Populations and Counterfactual Frequencies in Evolutionary Theory
}

\author{
Marshall Abrams \\ Department of Philosophy \\ George Washington University
}

\begin{abstract}
One finds intertwined with ideas at the core of evolutionary theory claims about frequencies in counterfactual and infinitely large populations of organisms, as well as in sets of populations of organisms. One also finds claims about frequencies in counterfactual and infinitely large populations - of events - at the core of an answer to a question concerning the foundations of evolutionary theory. The question is this: To what do the numerical probabilities found throughout evolutionary theory correspond? The answer in question says that evolutionary probabilities are "hypothetical frequencies" (including what are sometimes called "long-run frequencies" and "long-run propensities"). In this paper, I review two arguments against hypothetical frequencies. The arguments have implications for the interpretation of evolutionary probabilities, but more importantly, they seem to raise problems for biologists' claims about frequencies in counterfactual or infinite populations of organisms and sets of populations of organisms. I argue that when properly understood, claims about frequencies in large and infinite populations of organisms and sets of populations are not threatened by the arguments. Seeing why gives us a clearer understanding of the nature of counterfactual and infinite population claims and probability in evolutionary theory.
\end{abstract}

Preprint submitted to Elsevier Science

27 November 2005 
Key words: Population, Drift, Fitness, Probability, Frequency, Propensity

\section{Introduction}

On one hand, claims about frequencies in counterfactual and infinitely large populations of organisms or sets of populations lie at the core of evolutionary theory. On another, claims about frequencies in counterfactual and infinitely large populations of events lie at the core of an answer to a question concerning the foundations of evolutionary theory: What do the numerical probabilities found throughout evolutionary theory correspond to in the world? One not uncommon answer $^{1}$ is that many of the probabilities mentioned in evolutionary models are what are called "hypothetical frequencies". I intend this term to cover what are known as "long-run propensities" and sometimes "long-run frequencies". According to a hypothetical frequency interpretation of probability, ${ }^{2}$ probability can be defined in terms of a large, perhaps infinite, coun-

Email addresses: mars@gwu.edu, marshall@logical.net (Marshall Abrams). $U R L:$ http://members.logical.net/ marshall (Marshall Abrams).

1 I encounter it more often in conversation than in print.

2 An "interpretation of probability" is an account of what it is about the world that we are talking about in some context when we talk of probability. See (Eagle, 2004) or (Hájek, 2003) for a more detailed discussion of the term. Other well-known interpretations of probability include actual finite frequency, single-case propensity, subjective probability, logical probability, and classical probability. For critical overviews of many of the available alternatives see (Cohen, 1989; Eagle, 2004; Gillies, 2000a,b; Hájek, 2003; Howson and Urbach, 1993). Also note (Brandon, 1990; Millstein, 2003; Richardson and Burian, 1992; Sober, 2000), which discuss various interpretations of probability in relation to biology. There have been recent suggestions 
terfactual sequence of events $(\S 2)$. There are a number of objections to such theories, and I believe that the idea should at best be deployed only with extreme reservations. In this paper I review two of the most damning arguments against hypothetical frequency, the "unjustified counterfactuals problem" (§3) and "the reference sequence problem" (§5). These arguments are not as well known as they should be among those interested in philosophical issues concerning biology, and the arguments should give pause to anyone who wants to countenance hypothetical frequency as an interpretation of probability in evolutionary theory.

The real point of examining the two arguments, however, is to investigate whether - apart from the viability of hypothetical frequency - similar arguments are troublesome for common claims in evolutionary theory concerning counterfactual sets of populations and infinitely large populations. I explain why, when properly understood, these claims from evolutionary theory are not undermined by the arguments against hypothetical frequency. I'll look at two particular examples of such claims which seem representative of many similar ones. In Section 4 I examine a discussion from a well-known textbook (Roughgarden, 1979) which invokes counterfactuals about frequencies of states in sets of populations. I argue that a variant of the unjustified counterfactuals argument implies that these counterfactuals about frequencies of states in sets of populations are literally false. However, I explain that such

that biological probabilities might be understood in terms of ideas from statistical mechanics (Bouchard and Rosenberg, 2004; Rosenberg, 2001; Weber, 2001), in terms of complex causal structure and large scale patterns of events (Abrams, 2005), in terms of persistence of frequencies (Millikan, 2000), or in terms of theoretical role (Sober, 2000, 2005). 
counterfactual claims are merely stand-ins for other claims about probabilities concerning future states of a single population, and that the latter claims are not subject to anything like the unjustified counterfactuals problem (nor the reference sequence problem). In Section 6 I analyze an extension of a simple theorem about the decay of heterozygosity (Gillespie, 1998) in order to clarify the nature of claims about infinite populations of organisms. I argue that the character of the argument for the extended theorem allows it to avoid both the reference sequence problem and the unjustified counterfactuals problem.

\section{What is hypothetical frequency?}

First, let me illustrate very briefly the need for an interpretation of probability in a biological context where this need has been widely recognized. The propensity interpretation of fitness defines "fitness" so that it will be proportional to expected number of offspring (Brandon, 1978; Mills and Beatty, 1979). That is, according to the PIF, fitness is proportional to

$$
\sum_{n=0}^{\infty} n \times \mathrm{P}(O=n)
$$

where $\mathrm{P}(O=n)$ is the probability that a given organism or kind of organism will have $n$ offspring. There is then a further question as to what the probabilities $\mathrm{P}(O=n)$ consist in. 
To see the problem more clearly, note that mathematical axioms determine the formal characteristics of probability. ${ }^{3}$ The axioms justify inferences from statements about probabilities to statements about other probabilities or properties defined in terms of probability (variance, for example). The axioms themselves, however, do not determine what it is that statements mentioning probabilities literally assert about the world. The axioms don't specify what it is in the world, if anything, that numerical values of probability correspond to.

A hypothetical frequency interpretation of probability, or hypothetical frequency theory, is a proposal that at least some probabilities should be defined partly in terms of what frequencies in a very long sequence of events would be were such a sequence to occur. Thus a hypothetical frequency theory defines a kind of probability in terms of counterfactuals about relative frequencies in long sequences of trials. ${ }^{4}$ The following is then definitionally necessary for there to be a probability $r$ of an outcome $E$ : Were there many tokens of a particular kind of trial, the relative frequency of $E$ among all such trials would tend to $r$ in the limit or perhaps the finite long run. Note that it's crucial $\overline{3 \text { Here }}$ is one version of standard axioms that a probability function $\mathrm{P}(x)$ must satisfy:

(1) $0 \leq \mathrm{P}(x) \leq 1$

(2) $\mathrm{P}(x)+\mathrm{P}(y)=\mathrm{P}(x$ or $y)-\mathrm{P}(x$ and $y)$

These axioms are supplemented with axioms which specify how the events or propositions which are arguments to $\mathrm{P}(x)$ are allowed to combine. Axiom (2) is often replaced with a stronger axiom that covers infinite sums.

4 (Hacking, 1965) and (Gillies, 1973) are two classic statements of hypothetical frequency theories. See also note 6 . 
that there be specific circumstances which are repeated in every trial - that is what is meant by "kind of trial".

Hypothetical frequencies used as a basis for fitness, for example, would depend on counterfactuals concerning repetitions of trials consisting of a given organism's life in a given environment, or repetitions of trials consisting of lives of different organisms with the same genotype (or phenotype) in the same environment. For example, on a hypothetical limiting frequency account, the probability of a certain number of offspring would be defined as the limit, as the number of trials is increased, of the relative frequencies of trials in which exactly that number of offspring were produced. ${ }^{5}$ Notice that frequencies in a sequence of events as it becomes longer and longer are, properly speaking, frequencies in a sequence of larger and larger sets of events, where each succeeding set includes its predecessor as a proper subset.

I won't need to distinguish here between various kinds of hypothetical frequency theories, some of which go by the name "long-run propensity theory", while others go by "long-run frequency theory" or "limiting frequency theory". ${ }^{6}$ In the rest of the paper, I'll reserve "long-run frequency" for any fre-

$\overline{5}$ More precisely, let $\chi_{n}(i)$ be the function which is equal to 1 if organism or type $O$ 's number of offspring is $n$ on trial $i$, and equal to 0 otherwise. Then the relative frequency of cases in which $O$ has $n$ offspring in the first $k$ trials is $\sum_{i=0}^{k} \chi_{n}(i) / k$. The probability of having $n$ offspring would then be $\lim _{k \rightarrow \infty} \frac{1}{k} \sum_{i=0}^{k} \chi_{n}(i)$, where most of the trials which determine the values of $\chi_{n}(i)$ are to be counterfactual.

6 "Long-run propensity" is usually used for a theory which attributes objective probabilities to single trials but defines those probabilities in terms of counterfactuals about long sequences (cf. (Fetzer, 1981; Gillies, 1973, 2000b; Kyburg, 1974)). "Long-run frequency", by contrast, is somewhat more likely to be used for theories 
quency or limit of frequencies in a large number of trials, whether actual or counterfactual. Thus a hypothetical frequency theory will be any interpretation of probability given in terms of counterfactuals about long-run frequencies.

Hypothetical frequency seems to be on unusually shaky ground despite some continued popularity (e.g. in (Gillies, 2000b)). In Sections 3 and 5 I'll describe two important objections in detail . Both of the objections seem to have been rarely noticed in discussions of probability in evolutionary biology, and they have never to my knowledge been discussed as supplying more direct challenges to talk of counterfactual sets of populations and infinite populations $(\S \S 4,6){ }^{7}$

which apply only to fairly long sequences of actual trials; long-run frequency would then be defined in terms of the counterfactual extension of the sequence to many more trials. (It's generally agreed that "long-run frequency theory" and "limiting frequency theory" should be applied to the interpretations of von Mises (1957) and Reichenbach (1949), but views differ about whether these authors' views should be interpreted as invoking counterfactuals.) Long-run propensity in the sense mentioned is also contrasted with single-case propensity (which need not depend on counterfactual sequences). Both senses of "propensity" derive from (Popper, 1957, 1959). Note that single-case propensity is what philosophers of biology often mean by "propensity" — as in (Mills and Beatty, 1979) or (Brandon, 1990). Philosophers of biology tend to use "long-run frequency" for what I'm calling hypothetical frequency (Brandon, 1990; Graves et al., 1999; Rosenberg, 2001; Sober, 2000, 2005).

7 Works on philosophy of biology which mention objections relevant to hypothetical frequency include (Bouchard and Rosenberg, 2004; Graves et al., 1999; Richardson and Burian, 1992; Sober, 1984b, 2000). Other arguments counting against hypothetical frequency theories can be found in (Armstrong, 1983; Cohen, 1989; Eagle, 2004; Fetzer, 1981; Giere, 1976; Hájek, 1996, 2002; Howson and Urbach, 1993; Kyburg, 


\section{The unjustified counterfactuals problem}

Hypothetical frequency is defined in terms of counterfactuals about long-run frequencies in repetitions of similar trials. However, it is not at all clear that such counterfactuals are ever true (whether or not counterfactuals are problematic in general). I'll begin by considering a claim that a coin has a probability of $r$ to land heads. We could instead consider a claim about the probability for an organism to live to the age of reproduction, or to have 14 offspring rather than some other number. Intuitions will be clearer, however, if we step back from the complexities of biological contexts for the moment.

According to a simple hypothetical frequency theory, part of what makes it true that the probability of heads is $r$ is that if a coin were tossed many times, perhaps an infinite number of times, the limiting frequency of heads among the counterfactual tosses would be $r$, or would be about $r$, or the frequencies would in some sense or another tend toward $r$. There is insufficient reason to think that any such counterfactual is true, however. Note, first, that on every toss heads is a possibility. Thus it's possible to get heads as many times in a row as you like, even an infinite number of times. Any sequence of heads and tails is possible. A sequence with no limiting frequency is possible, and various limiting frequencies are possible. Why should we think that a counterfactual limiting frequency will have a single precise value (or even a narrow range of values)? An advocate of hypothetical frequency ought to provide a reason to think that counterfactual frequencies are determinate and will accord with what probabilities are supposed to be - to provide a reason to think that worlds with a long-run frequency of $r$ for heads are nearest, if you like (cf. (Lewis,

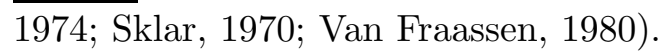


1973)). However, this issue is generally ignored. ${ }^{8}$

The preceding is an argument that even if there were a sequence of trials of the same kind, counterfactuals about frequencies would not be justified. Biological cases might be even more problematic, though, for it will often be unclear what a counterfactual situation with many similar biological trials would be like. As Richardson and Burian (1992) point out, actual environments may fluctuate in drastic ways over time. In that case, a counterfactual situation in which fairly similar conditions are repeated over and over again during a very long period of time may be particularly unlike real biological situations, requiring bizarre assumptions which make counterfactuals about repetitions impossible to evaluate.

Now, in the coin-tossing example, of course we feel that we won't see certain sequences, say, all heads in ten thousand trials. You might even be tempted to say that such sequences would not be likely to occur. However, that would simply be to say that the sequence is not probable, and you would then seem to need an independent source for probabilities over sequences of outcomes. One might think we could just require that there be some distribution of limit-

$\overline{\text { Eagle }}(2004)$ calls the unjustified counterfactuals problem the "Jeffrey problem" after a related argument in (Jeffrey, 1977). Variants of the argument can be found in (Armstrong, 1983; Giere, 1976; Hájek, 2002; Sklar, 1970). Sober's (1984b, p. 111; 2000, p. 62; 2005) version of this argument against hypothetical frequency assumes that we already have some (other) kind of objective probability on individual trials; part of the point of a hypothetical frequency theory is to be able to deny such assumptions. Note that van Fraassen (1980, ch. 6) "avoids" this problem by in effect simply stipulating that relative frequencies are the same in each relevant 'counterfactual' world - actually a structure in a model. 
ing frequencies in the relevant counterfactual worlds - that long-run frequency have the same value in almost every relevant world or that such and such proportion of worlds have limiting frequencies near some value. But we can't just count worlds; since physical properties such as location vary continuously, the number of relevant counterfactual worlds is uncountably infinite (cf. (Lewis, 1973, p. 20)). The complexity of biological environments makes this point particularly salient. So we would need to weight sets of worlds in some other way than by counting. That would be tantamount to providing a probability measure over these worlds, and this would again need an independent source. ${ }^{9}$

\section{Counterfactual sets of populations of organisms}

Biologists often make claims about frequencies in apparently counterfactual, large populations, sets of populations, etc. For example, Roughgarden's (1979, Ch. 5) discussion of genetic drift makes a number of claims about sets of populations of organisms. Each population can be in a state corresponding to a gene frequency, and Roughgarden investigates how many populations in a set will be in various states after a certain number of generations. Roughgarden gives examples using a set of laboratory populations of Drosophila melanogaster and using populations simulated on a computer. However, my interest is in Roughgarden's claims about frequencies of states in sets of counterfactual populations of organisms. Above, I reviewed an argument that hypothetical frequency's assumption that counterfactual frequencies are determinate is unjustified. Is there a similar problem for Roughgarden's claims about counterfactual sets of populations? Yes, I'll argue, if her statements are taken at

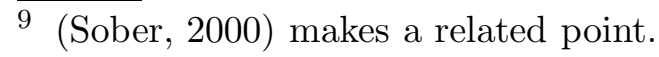


face value. However, I'll also argue that what Roughgarden actually intends to convey is not subject to an unjustified counterfactuals problem.

Roughgarden gives a simple example involving a counterfactual set of populations, for which "We can ... predict the fraction of the populations in each state through time." (p. 61) A small part of Roughgarden's argument for this claim will illustrate the issues involved. We imagine a set of populations, each with a fixed population size of 1 organism. The state of each such population is the number of $A$ alleles in its one member. Thus, a population in state 1 consists of an organism with one $A$ allele and one $a$ allele. Roughgarden gives the following argument:

... the probability of drawing two $A$ gametes [from those produced by a population in state 1] is $\frac{1}{4}$, of drawing an $A$ gamete and an a gamete is $\frac{1}{2}$ (i.e., $2 p q$ ), and of drawing two $a$ gametes is $\frac{1}{4}$. Therefore, $\frac{1}{4}$ of the populations in state 1 will move to state $0, \frac{1}{2}$ will stay in state 1 , and $\frac{1}{4}$ will move to state 2. (Roughgarden, 1979, p. 61) ${ }^{10}$

Notice that the argument's premise gives probabilities of various combinations of gametes into zygotes, while its conclusion gives frequencies of populations in various states at a later time. These frequencies are equal to the probabilities mentioned in the premise. Given that the conclusion claims that frequencies definitely would have certain values in a set of counterfactual populations, it's reasonable to ask whether an argument similar to that given against hypothetical frequency in the preceding section could be used to challenge Rough-

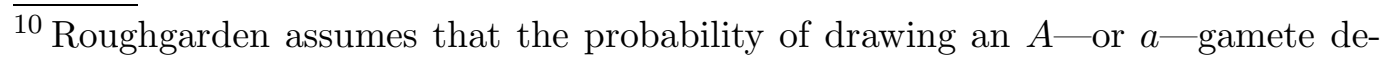
pends only on the number of alleles in the one parent. This corresponds to sampling with replacement.
} 
garden's conclusion.

First, notice one very significant difference between Roughgarden's conclusion and the claims which ground a hypothetical frequency theory of probability: While Roughgarden's conclusion is supposed to be justified by probabilities which are antecedently assumed to exist, hypothetical frequency theories generally make no assumption that any other kind of probability exists (since the point is to define a sense of probability in terms of something more basic). ${ }^{11}$ That hypothetical frequency is not based on any other kind of probability is part of what leaves counterfactual claims about long-run frequencies without any mooring. However, Roughgarden's claims about counterfactual frequencies are not in much better shape. For it simply doesn't follow from the fact that the probability of a kind of event is $r$ that the frequency of such events will be $r .{ }^{12}$ Roughgarden provides no additional premises which could justify

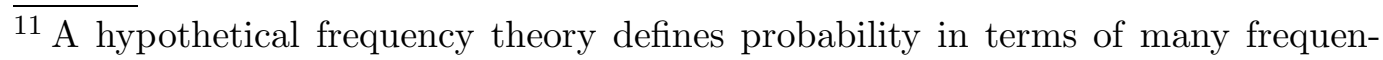
cies in finite sets, and frequencies are, mathematically, probabilities (they satisfy one standard set of axioms). But a hypothetical frequentist, while recognizing that fact, need not take the finite, counterfactual frequencies which she uses to define hypothetical frequency to be probabilities in sense of providing an interpretation of what is meant by "probability" in a particular scientific or practical context.

${ }^{12}$ A reviewer suggested that Roughgarden might be claiming only that the expected frequency of populations in a given state $s$ will be $r$. That would be to claim that the average of frequencies of populations in state $s$ will be $r$. Such an average would require either a set of sets of populations, or probabilities of a set of populations exhibiting various frequencies of populations in state $s$. While the latter probabilities could be derived from those mentioned in the premise of Roughgarden's argument, an overly elaborate interpretation of Roughgarden's conclusion would be required in either case. Roughgarden's direct language makes no use of "average", "expect", 
the inference, either. Compare: One cannot assume that if a fair coin were flipped 100 times, exactly 50 of the outcomes would be heads. Thus, like the hypothetical frequentist, Roughgarden's counterfactual claims that frequencies would have determinate values are not justified.

However, we should not take Roughgarden's claims about frequencies in sets of populations at face value. First, she notes that the predicted frequencies do not exactly match frequencies in computer simulations in two sets of 50 populations (p. 61). Yet a computer simulation of a set of populations would seem to capture what is essential to claims about counterfactual populations. Unlike an experiment using populations of real organisms, Roughgarden's simulations presumably include no observational errors or interfering effects that might cause an experiment to depart from predicted results. ${ }^{13}$ If such a simulation doesn't match what's supposed to occur in a counterfactual situation, there is either a problem with the simulation or a problem with claims about the counterfactual situation. Moreover, Roughgarden expresses no distrust of the results of the simulations. It seems reasonable to think that in the passages quoted above, Roughgarden did not really mean that the "predicted" frequencies of populations were what would in fact obtain in ideal counterfactual situations.

or "expectation". I suggest a simpler non-literal interpretation of Roughgarden's conclusions below.

13 The most interesting difference between a computer simulation and a realistic counterfactual case is that in the simulation, the stochasticity captured by an interpretation of probability in evolutionary theory is generally provided by a random number generating algorithm rather than whatever is the source of stochasticity in the world. Still, if the simulation is a good one, it should produce a pattern of behavior similar to what a counterfactual situation in the world would produce. 
Rather, Roughgarden's claims about counterfactual populations seem to be nothing more than a loose way of making concrete a claim concerning the probabilities of various states for any one population. A claim that the frequency of state $s$ in a counterfactual set of populations is $r$ is a pedagogical device for conveying the intuition that there is a probability of $r$ that a single population will change to state $s$. This is confirmed by other passages in Chapter 5, such as Roughgarden's remark that "we develop theory to predict the probability of the system's being in each possible state..." (p. 57, emphasis in original text) or a very similar remark in a footnote to page 61 .

Thus, Roughgarden's claims about counterfactual frequencies should not be read literally. Properly understood as claims about the probabilities for states of one population, they are strictly derivable from her assumptions about probabilities. (In the passage I quoted above, the "derived" probabilities of states simply are the probabilities mentioned in the premise within the quotation. Most of Roughgarden's inferences are not so direct.) Moreover, understood in this way Roughgarden's claims are not about frequencies in counterfactual populations (unless we were to give her probabilities an interpretation in terms of hypothetical frequency). Thus her claims are not subject to a version of the unjustified counterfactuals argument. ${ }^{14}$

\footnotetext{
$\overline{14}$ Most of Roughgarden's claims also are not subject to anything like the reference sequence problem, described below, because they only concern finite sets of populations. Roughgarden does make a passing remark about infinitely many populations (p. 61). It is not undermined by the reference sequence problem, for reasons illustrated below in Section 6. (Note that contrary to a remark Roughgarden makes, it is not strictly correct that "In the limit of infinitely many populations, each with the same finite number of individuals in it, the theory would be exact" (p. 61), i.e.
} 


\section{The reference sequence problem}

There is another problem for hypothetical frequency theories which invoke limiting frequencies based on infinite sequences of events rather than merely long finite sequences. As I'll show below, by changing the order of terms in such an infinite sequence we can make its associated limiting frequency take any value we want. We can even make the frequencies fail to converge to a limit. Hájek (2002) calls this the "reference sequence problem". ${ }^{15}$ The problem for a hypothetical frequency theory is this: Even if we had a reason to think that the limiting frequency for a sequence would be $r$ in a counterfactual situation, additional justification would be needed to take the particular order of those events to be the relevant order.

that her predictions would be correct for an infinitely large set of populations. In the limit as we increase the number of populations, the frequencies of populations in each state need not correspond exactly to the probabilities of those states. Rather, such an outcome has a probability of 1 ; that does not mean that it is guaranteed to occur. Consider for comparison an infinite sequence of tosses of a fair coin, where on each toss the probability of heads is .5. A strong law of large numbers implies that there is a probability of 1 that the limiting frequency of heads is .5 (i.e. the set of sequences with limit .5 has probability 1 ). Nevertheless, it is possible that an infinite sequence consisting of all heads will occur. In fact, that particular sequence has exactly the same probability as every other sequence, namely, a probability of zero. Similar remarks apply to many similar claims about infinite populations.)

15 This problem is often ignored in works which advocate hypothetical frequency as an interpretation of probability, and I don't believe that it has been widely recognized. It is not particularly new, however; a version can be found in (Russell, 1948, pp. 366f). 
First, note that it's often assumed that trials should be ordered by time. Why should time order be special, though? Consider, for example, hypothetical frequency as an interpretation of viability - probability of reaching reproductive age - in 17-year periodic cicadas. In the actual world, millions of cicadas begin their life cycles around the same time, and a population is dispersed over a wide area. What would a relevant counterfactual sequence of cicada lives look like? It should surely preserve the 17-year reproductive cycle, given that this cycle seems to be a strategy to reduce predation. If the counterfactual sequence of lives is to be ordered in time, does it involve one cicada every 17 years? Or, say, thousands or millions of cicadas ordered in time during one year, followed in the sequence by a similar number of cicadas 17 years later, and so on? Or are we to imagine an infinite temporal sequence of cicada lives within a single cycle? A sequence ordered spatially would seem to have at least as much intuitive sense as one laid out in time. That would require an additional choice of a particular spatial ordering, of course. In general, there does not seem to be any strong justification for any particular ordering of a counterfactual infinite sequence of biological trials. However, without a determinate order, the (counterfactual) limiting frequency will be indeterminate as well.

To see why, let us again look at a non-biological example. Notice that in an infinite sequence of heads and tails with limit $1 / 2$ for tails, there is an infinite number of heads and an infinite number of tails. Consider the tails outcome which occurs earliest in the sequence. Move the second of the tails outcomes into a new position just in front of that first tails outcome. Then move the third tails outcome into the place where the second was, and for every tails outcome that is moved up, move the next tails outcome into its 
place. We thus shift all of the tails outcomes without adding or removing any of them, creating a sequence in which there is one more tails outcome near the beginning of the sequence. Next repeat this whole procedure for each tails outcome after the initial pair of tails we just created. This results in a sequence of heads outcomes and pairs of tails outcomes, where a pair of tails replaces each single tails outcome in the original sequence. Thus the limiting frequency of tails pairs is $1 / 2$, but considered as a sequence of single outcomes, the limiting frequency of tails will now be $2 / 3$. However, nothing has been added or removed from the set of outcomes in the sequence.

The essential point here, which applies to biological trials as well as coin flips, is that in every infinite sequence in which an outcome has a limiting frequency other than 0 or 1 (as well as some with limit 0 or 1 ) there are exactly as many instances of the outcome and its contrary as in any other such sequence: Each set of instances is countably infinite. Thus any such sequence can be reordered into almost every other. The point, however, is not that we might devise a certain way of permuting an infinite sequence, but rather that a principled justification is needed for taking an infinite set of events in any particular order. Without that there is no principled reason for taking some particular limit as significant. ${ }^{16}$ (Note the point from Section 5, that a limit of frequencies is actually based on a sequence of sets of events, where each set

\footnotetext{
$\overline{16}$ Thus it does not matter that one could rule out the reordering example I gave by requiring that sequences satisfy a randomness requirement derived from von Mises' (1957) definition of probability. Any sequence with limit $r$ satisfying such a requirement can in principle be transformed into another satisfying the same requirement but with a different limit (or none), since all allowable sequences contain an infinite number of instances of each outcome.
} 
contains the sets earlier in the sequence. The present point then is that there is no principled reason for putting events in earlier, as opposed to only later sets in the sequence of sets.)

If biological probabilities such as those underlying fitness are to be given an interpretation as counterfactual limiting frequency, some rationale for taking counterfactual trials in some particular order is needed. However, I see no such rationale available for biological cases.

\section{Infinitely large populations of organisms}

Biologists sometimes make claims about infinitely large populations. For example, a common kind of claim is that in an infinitely large population, selection will drive a certain gene to fixation even though in a finite population drift may prevent that particular outcome. Talk of frequencies in an infinite population makes no sense, however, if interpreted literally. In an infinitely large population any property with a frequency other than zero would have to be shared by an infinite number of organisms. In that case we can't just take the quotient of two infinities to determine the frequency of the property. Generally, claims about infinite populations can only be justified as shorthands for claims about limits as population size is increased without bound. Note that such a limit involves a sequence of sets, each larger than the preceding, and recall from Section 5 that, strictly speaking, the sequence used to define hypothetical frequency is just such a sequence of sets. This suggests that an argument like the one at the core of the reference sequence problem might imply that there's something illegitimate about talk of infinite populations of organisms. It's instructive to see why this is not so. I'll give an overview of the 
reasons why followed by an illustration using a particular biological example.

First, note that the reference sequence problem-like the unjustified counterfactuals problem - is a problem for certain kinds of attempts to define a fundamental sense of objective probability in terms of limits of frequencies. It is not clear that any argument like it can raise problems for claims about limits where these claims are derived from assumptions about some other kind of probability which is assumed to have its own justification. Biologists' claims about infinite populations seem to be justified by limit theorems of this latter kind-ones justified by calculations over previously (sometimes tacitly) assumed probabilities. The resulting claims about infinite populations would seem to be problematic to whatever extent the assumed probabilities which justify them are problematic, but not because the claims concern infinitely large populations.

Second, the limits which justify infinite population claims don't seem to depend on the kind of facts that make the reference sequence problem applicable. The reference sequence problem is a problem for a limit defined in terms of a a sequence of sets. In particular, the reference sequence problem arises when there is inadequate justification for grouping items into these various sets. However, the sort of limits used to justify infinite population claims do not generally depend on which organisms are in which populations in a sequence of populations. What matters for the limit in such cases is only that the population size increases from one set in the sequence to the next (while, for example, gene frequencies remain the same). An extension of a simple theorem in (Gillespie, 1998, §2.2) will illustrate these points.

Gillespie derives the proposition that after $t$ generations, the probability $\mathcal{H}_{t}$ 
that two alleles drawn randomly (without replacement) are different is

$$
\mathcal{H}_{t}=\mathcal{H}_{0}\left(1-\frac{1}{2 N}\right)^{t}
$$

Here $\mathcal{H}_{0}$ is the corresponding initial probability and $N$ is the population size. Gillespie speaks only of large, not infinite populations, but this is nevertheless a simple illustration of the kind of proposition which biologists use to justify claims about infinite populations. Equation (1) implies that if the population size $N$ is infinite, the probability that two randomly chosen alleles are type identical is 1 , and further that this probability does not change with time. More precisely, this is a case where a claim about an infinite population is a shorthand for a claim about a limit as the population size is increased without bound. In equation (1), if $N$ is increased without bound, $\frac{1}{2 N}$ goes to 0 in the limit. Thus in the limit of population size, $\mathcal{H}_{t}$ remains constant at $\mathcal{H}_{0}$ :

$$
\lim _{N \rightarrow \infty} \mathcal{H}_{t}=\lim _{N \rightarrow \infty} \mathcal{H}_{0}\left(1-\frac{1}{2 N}\right)^{t}=\mathcal{H}_{0} \times(1-0)^{t}=\mathcal{H}_{0}
$$

First note that proposition (2) depends on assumptions about existing probabilities. Gillespie's derivation of equation (1) begins with a stated assumption that mating is random (p. 23). Gillespie defines "random mating" as what occurs when "mates are chosen with complete ignorance of their genotype (at the locus under consideration), degree of relationship, or geographic locality." (p. 11) It's common to use similar wording to imply that mating is random in the mathematical sense that every organism in the population has an equal probability of mating with any other. ${ }^{17}$ Indeed, when Gillespie uses the as$\overline{17}$ To say that there is an equal probability of choosing any individual is to attribute quite a bit of systematicity to outcomes of choices; it is to say that outcomes accord with the probability calculus. Strictly speaking, the mere claim that choices are 
sumption of random mating on pp. 23f, its implication is that the probability of a particular token allele being chosen (with replacement, presumably) to combine with another allele is $\frac{1}{2 N}$, and it is this implication which gives rise to the term subtracted from 1 in equations (1) and (2). This assumption about the probability of one allele combining with another would most naturally be justified by an assumption that each token allele present in the previous generation has an equal probability of being matched with another. Thus the implications of Gillespie's arguments for large population sizes $N$ are justified by a prior assumption that there are certain probabilities of alleles being combined into a new individual.

Second, notice that the limit in (2) depends only on the increase in $N$, along with the initial probability $\mathcal{H}_{0}$ of randomly chosen alleles being identical. It's not required that populations further on in the sequence contain earlier ones (as in a definition of "hypothetical frequency"). Altering which organisms first appear in earlier or later populations in the sequence cannot affect the limit, because the limit statement makes no reference to the members of the populations: The only variable element of the limit statement is the population size $N$. There is thus no reference sequence problem, because the details of the sequence do not matter. (Note that for the same reason, the unjustified counterfactuals problem does not apply here. Counterfactuals about a sequence of populations are not relevant to the limit because what matters in the mathematical sequence is simply population size.)

It is reasonable to think that other claims concerning infinite populations made "with complete ignorance" of a property is consistent with choices being so erratic that there is no sense in which outcomes can be characterized in terms of probability. 
have similar properties. In that case these claims also would not be subject to anything like the reference sequence problem.

\section{Conclusion}

The primary purpose of this paper was to investigate whether two objections to hypothetical frequency interpretations of probability also tell against claims in evolutionary theory concerning counterfactual, possibly infinite populations and sets of populations. A secondary purpose was to argue that hypothetical frequency interpretations should not be taken seriously as candidates for an interpretation of probabilities in evolutionary theory. After presenting each of the two objections to hypothetical frequency theories, I discussed common claims in evolutionary theory which plausibly would be vulnerable to similar objections. I then argued that in each case the evolutionary claims turn out in the end to be safe from the objections. Examples from two textbooks (Roughgarden, 1979; Gillespie, 1998) illustrated the relevant biological claims.

The unjustified counterfactuals problem is the problem of providing an independent justification of counterfactual claims about long-run frequencies. It appears to be intractable. We saw that counterfactual claims like Roughgarden's (1979, Ch. 5) about frequencies of populations in various states are in fact literally false, for reasons similar to those elaborated in the unjustified counterfactuals argument. Thus, strictly speaking, such claims do have an "unjustified counterfactuals problem". However, I argued that Roughgarden's counterfactual claims should not be interpreted literally. Understood as claims about probabilities of states of a single population, the claims are not subject to an unjustified counterfactuals problem. Such claims about probabilities are 
derived from assumptions about the existence of other probabilities.

The reference sequence problem is the problem that in a limit of frequencies in an infinite sequence of events, there does not seem to be a principled justification for taking the events in a particular order. Yet changing their order can result in a different limiting frequency. Using an extension of a simple theorem about heterozygosity presented by Gillespie (1998, §2.2), I argued that the limits which ground infinite population claims do not depend on ordering of elements in a way which would make something like the reference sequence problem applicable.

It is thus reasonable to think that counterfactual claims about large and infinite populations and sets of populations are not undermined by the two arguments against hypothetical frequency. However, the discussion of the example from Roughgarden shows the importance of properly interpreting biologists' claims about probability and frequency.

Note that in both of the examples I gave, the claims made by biologists were justified by derivation from assumptions that certain probabilities exist. These probabilities were not simply the probabilities of having certain numbers of offspring which are often mentioned in discussions of biological fitness. Thus my discussion helps to emphasize the need for an interpretation or interpretations of probability to make sense of various kinds of probabilities assumed in evolutionary theory. ${ }^{18}$ For example, an interpretation of probability is needed to provide a foundation for models in which there is or is not assortative mating, or for models in which the probability of migration enters into calculations. Without understanding what interpretation of probability grounds

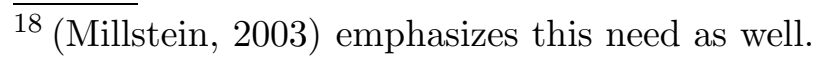


claims about populations and evolution, it is just not clear what is being asserted. $^{19}$

\section{Acknowledgements}

I'm grateful for feedback from Murat Aydede, Robert Brandon, Patrick Forber, Stuart Glennan, Ruth Millikan, Alex Rosenberg, Elliott Sober, William C. Wimsatt, and an anonymous reviewer.

\section{References}

Abrams, M., 2005. The causal structure of biological fitness, MS.

Armstrong, D., 1983. What is a Law of Nature? Cambridge University Press.

Bouchard, F., Rosenberg, A., 2004. Fitness, probability, and the principles of natural selection. British Journal for the Philosophy of Science 55 (4), 693-712.

Brandon, R. N., 1978. Adaptation and evolutionary theory. Studies in the History and Philosophy of Science 9 (3), 181-206, reprinted in (Sober, 1984a).

Brandon, R. N., 1990. Adaptation and Environment. Princeton University.

Cohen, L. J., 1989. An Introduction to the Philosophy of Induction and Probability. Oxford University Press.

Eagle, A., 2004. Twenty-one arguments against propensity analyses of probability. Erkenntnis 60, 371-416.

Fetzer, J. H., 1981. Scientific Knowledge: Causation, Explanation, and Corroboration. Boston Studies in the Philosophy of Science. D. Reidel.

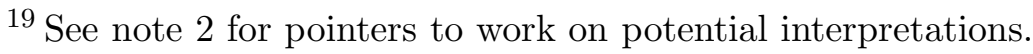


Giere, R. N., 1976. A Laplacean formal semantics for single-case propensities. Journal of Philosophical Logic 5, 321-353.

Gillespie, J. H., 1998. Population Genetics: A Concise Guide. The Johns Hopkins University Press.

Gillies, D. A., 1973. An Objective Theory of Probability. Methuen \& Co. Ltd.

Gillies, D. A., 2000a. Philosophical Theories of Probability. Routledge.

Gillies, D. A., 2000b. Varieties of propensity. British Journal for the Philosophy of Science 51, 807-835.

Graves, L., Horan, B. L., Rosenberg, A., 1999. Is indeterminism the source of the statistical character of evolutionary theory? Philosophy of Science 66, $140-157$.

Hacking, I., 1965. The Logic of Statistical Inference. Cambridge University Press.

Hájek, A., 1996. 'Mises Redux'-redux: Fifteen arguments against finite frequentism. Erkenntnis 45, 209-227.

Hájek, A., 2002. Fifteen arguments against hypothetical frequentism, MS.

Hájek, A., 2003. Interpretations of probability. In: The Stanford Encyclopedia of Philosophy. Http://plato.stanford.edu/archives/sum2003/entries/probabilityinterpret/.

Howson, C., Urbach, P., 1993. Scientific Reasoning: The Bayesian Approach, 2nd Edition. Open Court.

Jeffrey, R. C., 1977. Mises redux. In: Butts, R. E., Hintikka, J. (Eds.), Basic Problems in Methodology and Linguistics. D. Reidel.

Kyburg, Jr., H. E., 1974. Propensities and probabilities. British Journal for the Philosophy of Science 25 (4), 358-375, reprinted with a correction in (Tuomela, 1978). 
Lewis, D., 1973. Counterfactuals. Harvard University Press.

Millikan, R. G., 2000. What has natural information to do with intentional representation? In: On Clear and Confused Ideas. Cambridge University Press, pp. 217-237.

Mills, S., Beatty, J., 1979. The propensity interpretation of fitness. Philosophy of Science 46 (2), 263-286, reprinted in (Sober, 1984a) and (Sober, 1994).

Millstein, R. L., 2003. Interpretations of probability in evolutionary theory. Philosophy of Science 70, 1317-1328.

Popper, K. R., 1957. The propensity interpretation of the calculus of probability, and the quantum theory. In: S. Körner (Ed.), Observation and Interpretation. Academic Press Inc., Butterworths Scientific Publications, pp. $65-70$.

Popper, K. R., 1959. The propensity interpretation of probability. British Journal for the Philosophy of Science 10, 25-42.

Reichenbach, H., 1949. The Theory of Probability. University of California.

Richardson, R. C., Burian, R. M., 1992. A defense of propensity interpretations of fitness. In: PSA 1992. Vol. 1. Philosophy of Science Association, pp. 349362.

Rosenberg, A., 2001. Discussion note: Indeterminism, probability, and randomness in evolutionary theory. Philosophy of Science 68, 536-544.

Roughgarden, J., 1979. Theory of Population Genetics and Evolutionary Ecology: An Introduction. Macmillan.

Russell, B., 1948. Human Knowledge: Its Scope and Limits. Simon and Schuster.

Sklar, L., 1970. Is probability a dispositional property? Journal of Philosophy $67(11), 355-366$.

Sober, E. (Ed.), 1984a. Conceptual Issues in Evolutionary Biology. MIT Press. 
Sober, E., 1984b. The Nature of Selection. MIT Press.

Sober, E. (Ed.), 1994. Conceptual Issues in Evolutionary Biology, 2nd ed. MIT Press.

Sober, E., 2000. Philosophy of Biology, 2nd Edition. Westview Press.

Sober, E., 2005. Evolutionary theory and the reality of macro probabilities. In:

Eells, E., Fetzer, J. (Eds.), Probability in Science. Open Court, forthcoming. Tuomela, R. (Ed.), 1978. Dispositions. D. Reidel.

Van Fraassen, B. C., 1980. The Scientific Image. Oxford University Press.

von Mises, R., 1957. Probability, Statistics and Truth. Dover.

Weber, M., 2001. Determinism, realism, and probability in evolutionary theory: The pitfalls, and how to avoid them. Philosophy of Science 68 (3), S213-S224. 